\title{
A New Scheme to Enhance the Third-Harmonic Generation in Graphene
}

\author{
Jian Wei You ${ }^{1}$ and Nicolae C. Panoiu ${ }^{1}$ \\ ${ }^{1}$ Department of Electronic and Electrical Engineering, University College London, London, United Kingdom, WC1E 7JE \\ e-mail address: j.you@ucl.ac.uk and n.panoiu@ucl.ac.uk
}

\begin{abstract}
In this paper, a new approach is proposed to efficiently enhance the third-harmonic generation (THG) in graphene by employing a bilayer-grating structure. This scheme relies on the fact that the optical resonances of graphene structures are determined by the geometry. To boost the THG, we optimize the geometry of graphene bilayer gratings, thus achieving more than eight orders of magnitude enhancement of the THG intensity.
\end{abstract}

Keywords-Nonlinear optics; surface plasmons; third-harmonic generation; graphene

\section{INTRODUCTION}

In the past decade, graphene [1,2] has attracted increasing interest both in science and engineering, because of its unique and remarkable properties. Graphene has been widely used in photonic devices [3,4], as its physical properties [5,6] can be easily and ultrafast tuned via electric gating and chemical doping. In addition to applications to passive devices, its nonlinear optical properties have also been effectively employed to active nanophotonic devices [7-9], primarily because the local optical field can be strongly enhanced upon the generation of surface plasmons of graphene structures.

In this paper, we employ the geometry dependence of the resonance wavelength of plasmons in graphene gratings to demonstrate that giant enhancement of third-harmonic generation (THG) can be achieved in properly designed graphene bilayer gratings. In this novel type of optical devices, one graphene grating possesses an intrinsic double plasmon resonance, with additional THG enhancement being provided by the other grating, for which the wavelength of the fundamental plasmon is equal to a third of that of the fundamental plasmon of the first grating.

\section{STURCTURE AND OPTIMIZATION}

\section{A. Graphene Bilayer Gratings}

The schematic of the analyzed graphene bilayer grating is given in the inset in Fig. 1. Thus, two graphene gratings are placed on the top and bottom facets of a dielectric spacer with the relative permittivity $\epsilon_{s}=2.25$. The periods of the two gratings are $\Lambda_{1}=100 \mathrm{~nm}$ and $\Lambda_{2}=50 \mathrm{~nm}$. The widths of the graphene ribbons, $W_{1}$ and $W_{2}$, and the thickness, $H$, of the spacer are quantities we optimized for maximum THG enhancement.

\section{B. Optimization Procedure}

Using an in-house developed generalized-source FDTD method [13-15], we have first studied a single-layer graphene grating with $\Lambda_{1}=100 \mathrm{~nm}$, placed on top of a spacer with $H=50 \mathrm{~nm}$.
In our simulation, the Fermi energy of graphene is $0.6 \mathrm{eV}$, the relaxation time is $0.2 \mathrm{ps}$, and the temperature is $300 \mathrm{~K}$. Similarly to the approach described in [9], we first determined the optical absorption for different values of the width $W_{1}$ and found a double plasmon resonance of this single-layer graphene grating at $W_{1}=83 \mathrm{~nm}$ and wavelengths $\lambda_{0}=9.57 \mu \mathrm{m}$ and $\lambda_{0} / 3=$ $3.19 \mu \mathrm{m}$. Thus, this grating is resonant at both the fundamentalfrequency (FF) and third-harmonic (TH). Then, we varied the width $W_{2}$ of the graphene grating with period $\Lambda_{2}=50 \mathrm{~nm}$ and determined the width for which a first-order plasmon resonance exists at the wavelength $\lambda=\lambda_{0} / 3=3.19 \mu \mathrm{m}$. Guided by this analysis, we finally considered a graphene bilayer grating made of the two optimized graphene gratings placed on the two facets of the spacer, as per the inset shown in Fig. 1.

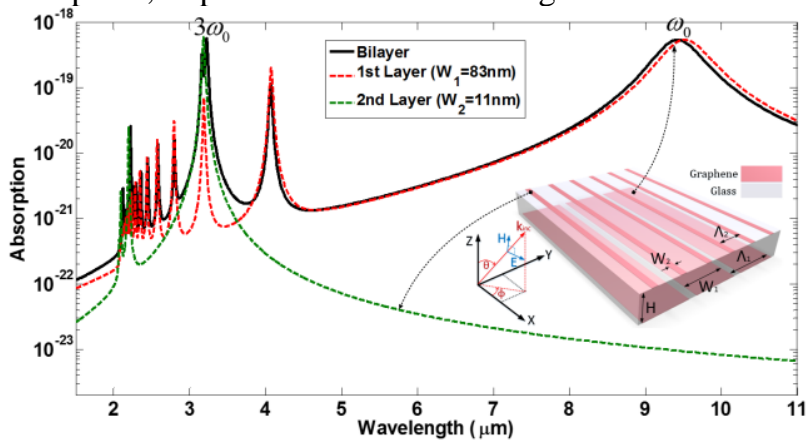

Fig. 1. Mechanism of third-harmonic generation (THG) enhancement in graphene bilayer gratings.

The strength of the local nonlinear current is determined by the electric near-field at the FF. Moreover, due to the plasmon resonance, the near-field in graphene gratings can be strongly enhanced at the plasmon resonance wavelengths. This is the origin of the strong peaks at $\lambda_{0}\left(\omega_{0}\right)$ and $\lambda_{0} / 3\left(3 \omega_{0}\right)$ in the spectrum plotted in Fig. 1 with a red dashed line. However, the resonance at $\mathrm{TH}$ frequency $3 \omega_{0}$ is rather weak, in spite of the fact that the grating has a double resonance. In order to further enhance the optical response at the $\mathrm{TH}$ frequency, we added another graphene grating specially designed to have a first-order resonance at $3 \omega_{0}$. However, when the second graphene layer is added, due to the mutual optical coupling between the two gratings, the double resonance condition is no longer fulfilled (see Fig. 1, black solid line). In order to restore the double resonance condition in the graphene bilayer grating, we slightly vary the width $W_{1}$ of the bottom graphene grating from $83 \mathrm{~nm}$ to $86 \mathrm{~nm}$. Finally, we varied the thickness $H$ of the spacer, and found that the double plasmon resonance condition is restored at the particular thickness of $H=22 \mathrm{~nm}$ of the graphene bilayer grating, as illustrated by the black solid line shown in Fig. 2. 


\section{RESULTS AND DISCUSSION}

\section{A. Linear Optical Response}

Using our generalized-source FDTD method, we calculated the linear response of a single-layer graphene grating and the optimized graphene bilayer grating. In this example, the parameters of the single-layer graphene grating are set to $W_{1}=$ $83 \mathrm{~nm}$ and $\Lambda_{1}=100 \mathrm{~nm}$. Furthermore, the parameters of the optimized graphene bilayer grating are found to be $\Lambda_{1}=$ $100 \mathrm{~nm}, \Lambda_{2}=50 \mathrm{~nm}, W_{1}=86 \mathrm{~nm}, W_{2}=11 \mathrm{~nm}$, and $H=$ $22 \mathrm{~nm}$. The optical absorption spectra shown in Fig. 2 clearly prove that the absorption in the graphene bilayer grating is enhanced as compared to that in a single-layer graphene grating when the double plasmon resonance condition is satisfied for both gratings. In particular, the linear absorption at the $\mathrm{TH}$ $\left(3 \omega_{0}\right)$ is enhanced by about 8 times.

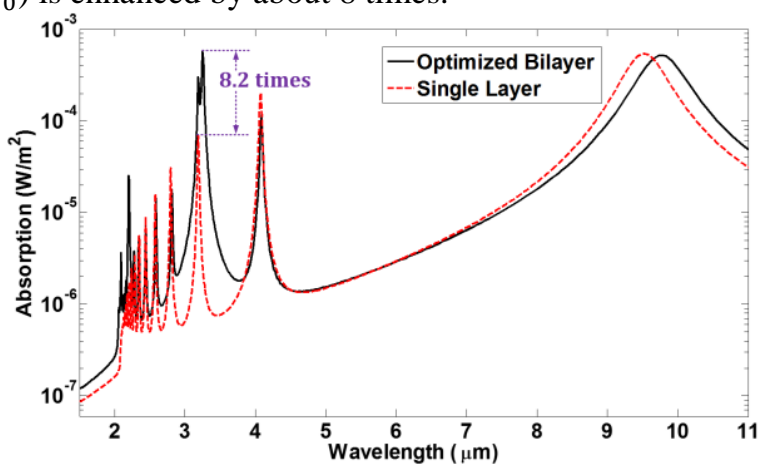

Fig. 2. Comparison of the linear response of the graphene bilayer gratings with a single-layer graphene grating.

\section{B. Plasmon Enhancement of the THG}

In order to demonstrate that THG can be enhanced by using the optimized graphene bilayer grating, we have calculated the $\mathrm{TH}$ radiation intensity by using our generalized-source FDTD method of a uniform graphene sheet, a single-layer graphene grating, designed to possess a double resonance, and the optimized graphene bilayer grating. The corresponding optical spectra at the TH are presented in Fig. 3.

The spectra shown in Fig. 3 clearly demonstrate that the TH radiation intensity of the optimized graphene bilayer grating is enhanced by about 44 times, as compared to the nonlinear optical response in the single-layer case. More importantly, as compared to a uniform graphene sheet, the THG intensity in the single and bilayer graphene gratings is enhanced by about six and eight orders of magnitude, respectively. This finding clearly proves the effectiveness of the double resonance mechanism in enhancing the THG.

A double plasmon resonance exists both in the single and bilayer graphene gratings. In particular, in both cases a localized surface graphene plasmon can be excited at $3 \omega_{0}$, if we pump either of the graphene gratings at $\omega_{0}$. However, as illustrated in Fig. 3, the bilayer graphene grating possesses a fundamental plasmon resonance at the $\mathrm{TH}$, whereas in the case of the single layer graphene grating the plasmon at the $\mathrm{TH}$ is a higher-order resonance, and this is in fact the main reason for the observed increase in the TH intensity.

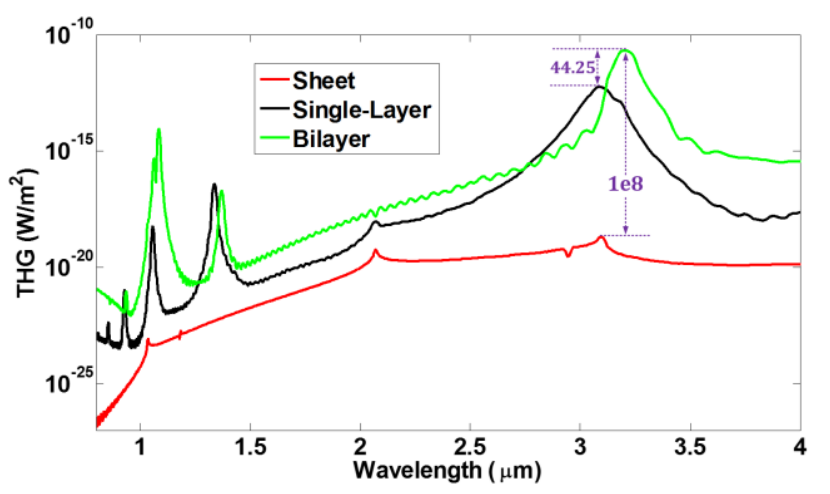

Fig. 3. Comparison of the nonlinear response between an optimized graphene bilayer grating with a graphene sheet and a single-layer graphene grating.

\section{CONCLUSION}

To summarize, we have demonstrated that giant enhancement of THG can be achieved in graphene multilayer gratings by simply tuning the spectral location of plasmon resonances.

\section{ACKNOWLEDGMENT}

This work was supported by the European Research Council, ERC Grant Agreement no. ERC-2014-CoG-648328.

\section{REFERENCES}

[1] K. S. Novoselov, A. K. Geim, S. V. Morozov, D. Jiang, Y. Zhang, S. V. Dubonos, etc al, "Electric field effect in atomically thin carbon films," Science, vol. 306, pp. 666-669, Oct 2004.

[2] K. S. Novoselov, A. K. Geim, S. V. Morozov, D. Jiang, M. I. Katsnelson, I. V. Grigorieva, etc al, "Two-dimensional gas of massless Dirac fermions in graphene," Nature, vol. 438, pp. 197-200, Nov 2005.

[3] F. Schwierz, "Graphene transistors," Nat. Nanotechnol. Vol. 5, pp. 487496, May 2010.

[4] R. R. Nair, P. Blake, A. N. Grigorenko, K. S. Novoselov, T. J. Booth, T. Stauber, etc al, "Fine structure constant defines visual transparency of graphene," Science, vol. 320, pp. 1308, June 2008.

[5] A. K. Geim, and K. S. Novoselov, "The rise of graphene," Nat. Mater, vol. 6, pp. 183-191, March 2007.

[6] A. A. Balandin, S. Ghosh, W. Bao, I. Calizo, D. Teweldebrhan, F. Miao, etc al, "Superior thermal conductivity of single-layer graphene," Nano Letters, vol. 8, ppp. 902-907, Feb 2008.

[7] M. T. Manzoni, I. Silveiro, F. J. G. Abajo, and D. E. Chang, "Secondorder quantum nonlinear optical processes in single graphene nanostructures and arrays," New J. Phys, vol. 17, art. no. 083031, Aug 2015.

[8] M. Weismann and N. C. Panoiu, "Theoretical and computational analysis of second- and third-harmonic generation in periodically patterned graphene and transition-metal dichalcogenide monolayers," Phys. Rev. B, vol. 94, art. no. 035435, July 2016.

[9] J. W. You, J. You, M. Weismann, and N. C. Panoiu, "Double-resonant enhancement of third-harmonic generation in graphene nanostructures," Phil. Trans. R. Soc. A, vol. 375, art. no. 20160313, Feb 2017.

[10] A. A. Shcherbakov and A. V. Tishchenko, "Fast numerical method for modeling one-dimensional diffraction gratings," Quantum Electronics, vol. 40, pp. 538-544, Aug 2010.

[11] M. Weismann, D. F. G. Gallagher, and N. C. Panoiu, "Nonlinear generalized source method for modeling second-harmonic generation in diffraction gratings," J. Opt. Soc. Am. B, vol. 32, pp. 523-533, March 2015.

[12] J. W. You, S. R. Tan and T. J. Cui, "Novel adaptive steady-state criteria for finite-difference time-domain method," IEEE Trans. Microwave Theory and Tech, vol. 12, pp. 2849-2858, Dec 2014 